\title{
A THICK ANGER CAMERA FOR GAMMA-RAY ASTRONOMY
}

W. R. Cook, M. Finger, and T. A. Prince

California Institute of Technology, Pasadena, CA 91125

\section{Abstract}

The NaI(Tl) Anger camera is a natural candidate for a position sensitive detector in imaging of astrophysical $\boldsymbol{\gamma}$-ray sources. Here we present laboratory measurements of the response of a relatively thick $(5.1 \mathrm{~cm}) \mathrm{NaI}(\mathrm{Tl})$ Anger camera designed for coded aperture imaging in the $50 \mathrm{keV}$ to $2 \mathrm{MeV}$ energy range. We obtained a position resolution of $10.5 \mathrm{~mm}$ FWHM at $122 \mathrm{keV}$ and $6.3 \mathrm{~mm}$ FWHM at $662 \mathrm{keV}$. The energy resolution was 7\% FWHM at 662 $\mathrm{keV}$. We discuss the ability of the detector to resolve the depth of the $\gamma$-ray interaction and the use of this depth resolution to reduce back-incident and internal background.

\section{Introduction}

Over the next decade $\gamma$-ray astronomy promises to move into an exciting new phase. As the flux sensitivity improves to levels expected for instruments of the Gamma Ray Observatory and beyond, the number of observable astrophysical sources in the 50 $\mathrm{keV}$ to $2 \mathrm{MeV}$ energy range will increase from dozens to over 1000 . Imaging capability will become essential, not only to avoid source confusion, but to provide accurate source locations and the capability to measure numerous sources simultaneously. Thus, we have undertaken to adapt coded aperture techniques to the particular needs of $\gamma$-ray astronomy.

The NaI(Tl) Anger camera was invented in $1957^{1}$ and has been extensively developed for medical imaging in the hard x-ray energy range. The Anger camera was thus a natural and economical choice as the position sensitive detector for our imaging studies and for use in a first generation $\gamma$-ray telescope. Our initial tests were made with a standard thin $(1.27 \mathrm{~cm}) \mathrm{NaI}(\mathrm{Tl})$ Anger camera and successfully demonstrated the imaging capabilities of rotating hexagonal-celled uniformly redundant arrays (HURAs) at hard xray energies. ${ }^{2}$ Here we report on the performance of a relatively thick $(5.1 \mathrm{~cm}) \mathrm{NaI}(\mathrm{Tl})$ Anger camera designed for $\gamma$-ray imaging at energies up to $\sim 2 \mathrm{MeV}$.

\section{The Detector and Instrumentation}

The $\mathrm{NaI}(\mathrm{Tl})$ detector was $40.6 \mathrm{~cm}$ in diameter by $5.1 \mathrm{~cm}$ thick and was manufactured of "Polyscin" material by Harshaw Chemical Co. The detector was mounted by Harshaw in a standard camera plate configuration with a $1.27 \mathrm{~cm}$ thick optical window. Nineteen $76 \mathrm{~mm}$ diameter photo-multiplier tubes (PMTs, type Hamamatsu 1307) were coupled directly to the optical window in a hexagonal array with center to center spacing of $8.25 \mathrm{~cm}$. The area of the optical window not covered by PMTs was covered by white cardboard.

The camera plate PMTs were instrumented to closely approximate the desired situation in which each PMT is individually pulse-height analyzed for each $\gamma$-ray event. The only deviation from such a scheme was required by the 15 input limitation of the available pulse-height analysis system. Twelve of the nineteen PMTs (0 through 10 plus 18 , as labeled in Figure 1) were individually pulse-height analyzed, while the remaining three input channels were used to analyze three sum signals: $(11+12),(13+14+15)$, and $(16+17)$. We then confined the majority of our measurements to
Figure 1. Response map taken with $\gamma$-ray beams from a collimated 662 $\mathrm{keV}$ source. The data were taken inside the area enclosed by the dashed line and were repeated using the detector symmetries to illustrate the size of the usable detector area in relation to the position resolution. (Before combining data from the separate beams, background events outside each beam spot were removed.)

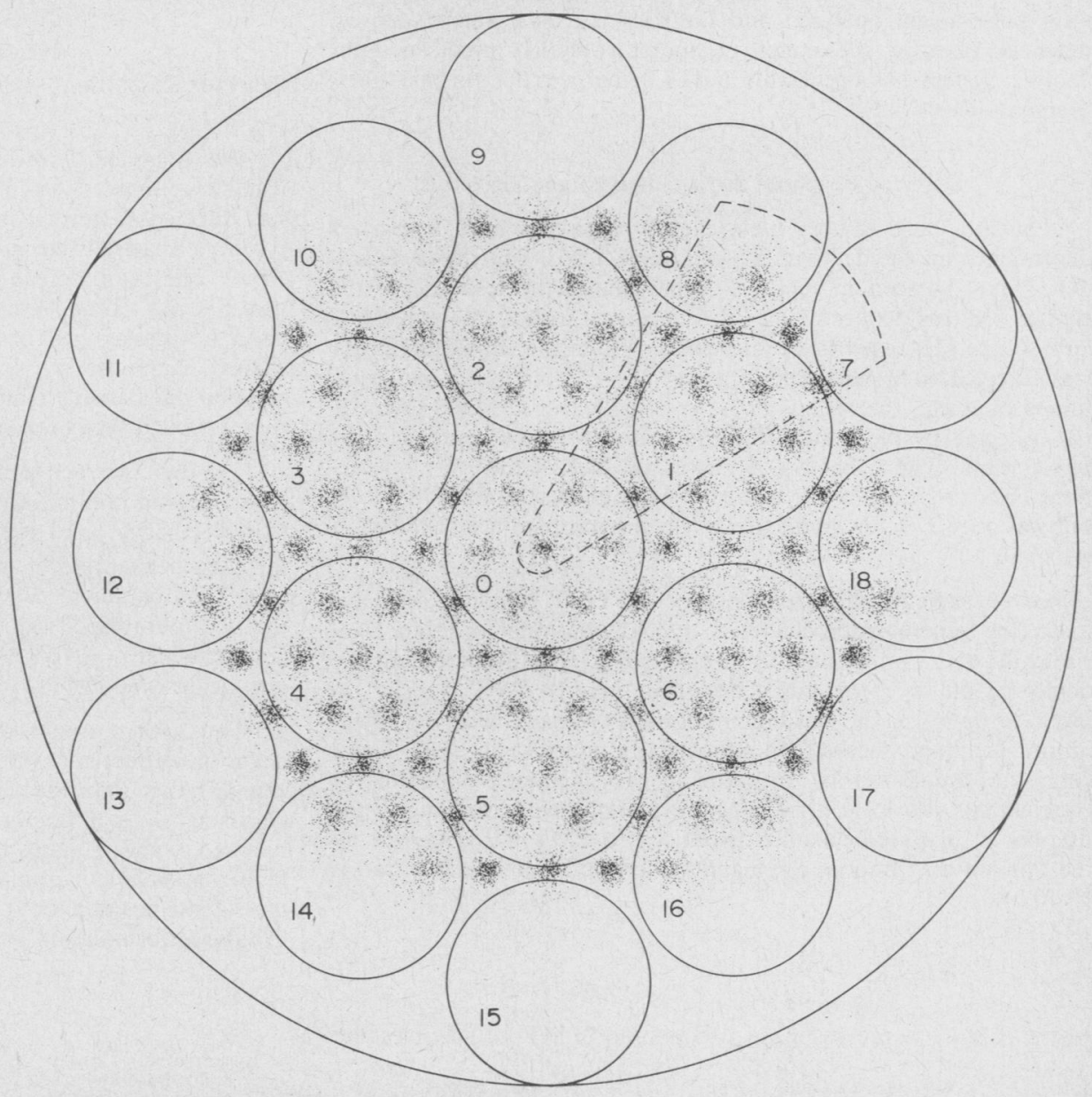

0018-9499/85/0002-0129\$01.00 @ 1985 IEEE 


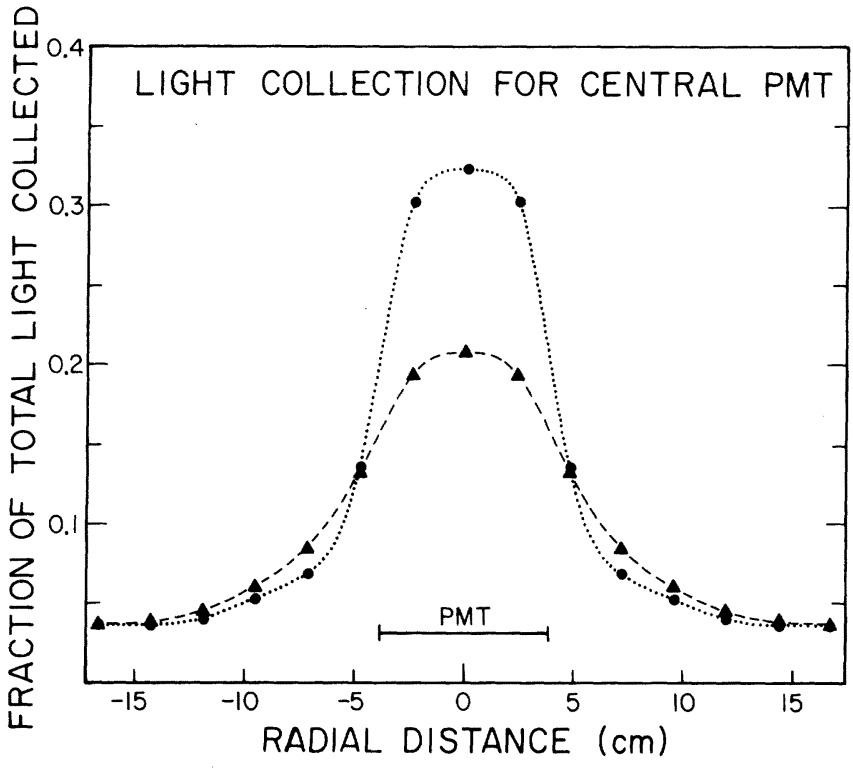

Figure 2. The response of the central PMT versus radial distance. The filled circles indicate the response due to $\gamma$-ray events occurring deep in the $\mathrm{NaI}(\mathrm{Tl})$ crystal near the surface close to the photo-multiplier tubes, while the solid triangles give the response due to events occurring near the $\mathrm{NaI}(\mathrm{Tl})$ front surface. (Points to the left of center were obtained by symmetry.)

the wedge-shaped region shown in Figure 1 so that the summed signals would be small and contain little of the relevant information on $\gamma$-ray interaction location. For each $\gamma$-ray event all 15 channels were pulse-height analyzed and the resulting data were stored on magnetic tape for subsequent computer study. A nineteen input analysis system has been built and is being used for further measurements.

\section{Detector Response and a 3-D Position Algorithm}

For the thick camera plate the PMT signals have a marked dependence on the depth of a $\gamma$-ray interaction. This depth dependence may be seen in Figure 2 which shows the response of the central PMT as deduced from measurements with a collimated 662 $\mathrm{keV}$ source (discussed in detail below). When the collimated beam was directed at normal incidence to the camera plate and positioned opposite the central PMT, the fraction of the total light signal collected by the central PMT increased with the depth of $\gamma$-ray penetration. The signal was a maximum of $32 \%$ for $\gamma$-ray interactions occurring deep in the camera plate and close to the PMT face, but was only $21 \%$ for interactions near the front surface of the camera plate.

Given such depth dependence of the PMT response, we have taken the general approach of a three-dimensional position algorithm for determining both the transverse $(x, y)$ coordinates and the depth (z) of the $\gamma$-ray interaction from the 19 PMT signals. In order to optimize the position resolution we have used the "maximum likelihood method" in which the interaction location $\vec{x}$ and energy $E$ are found by maximizing the likelihood function, or equivalently it's logarithm. Since the statistical variation of the number $n_{i}$ of photo-electrons produced in PMT $\mathrm{i}$ is governed by the Poisson distribution, the logarithm of the likelihood function is given by: ${ }^{3}$

$$
\ln L(\vec{x}, E)=\sum_{i}\left[n_{i} \ln N_{i}(\vec{x}, E)-N_{i}(\vec{x}, E)\right]+C,
$$

where $N_{i}(\vec{x}, E)$ is the response function for PMT $\mathrm{i}$ and gives the expected number of photo-electrons as a function of interaction location and $\gamma$-ray energy, and the function $C$ depends only on the $n_{i}$

The problem simplifies if we assume that the response functions are separable functions of $\vec{x}$ and $E$ :

$$
N_{i}(\vec{x}, E)=l(E) f_{i}(\vec{x}) .
$$

To a good approximation the PMT responses are proportional to $\gamma$-ray energy at a given $\vec{x}$. Small deviations from proportionality are due to the non-linearity of the $\mathrm{NaI}(\mathrm{Tl})$ light response and are accounted for by the function $l(E)$. for $E$ :

We may solve $\partial \ln L / \partial E=0$ for to obtain an implicit expression

$$
l(\dot{E})=\frac{\sum_{i} n_{i}}{\sum_{i} f_{i}(\vec{x})} .
$$

Upon substitution of [2] and [3] into equation [1] we find that $\ln L$ reduces within constant terms to:

$$
\ln \hat{L}(\vec{x})=\sum_{i}\left[\hat{n}_{i} \ln \hat{N}_{i}(\vec{x})-\hat{N}_{i}(\vec{x})\right]
$$

where the PMT responses $\hat{n}_{i}$ and the response functions $\hat{N}_{i}$ are now normalized:

$$
\hat{n}_{i} \equiv \frac{n_{i}}{\sum_{j} n_{j}}
$$

and

$$
\hat{N}_{i}(\vec{x}) \equiv \frac{N_{i}(\vec{x}, E)}{\sum_{j} N_{j}(\vec{x}, E)} .
$$

\section{Detector Response}

We obtained knowledge of the required response functions using a collimated $662 \mathrm{keV} \gamma$-ray source $\left({ }^{137} \mathrm{Cs}\right)$. The $\gamma$-ray beam was directed at normal incidence to the camera plate and could be positioned laterally to an accuracy of $0.1 \mathrm{~mm}$ by an $x-y$ translation stage. The beam spread at the center of the NaI(Tl) crystal was 3 mm FWHM. Data were obtained for source locations chosen to lie on a hexagonal grid of spacing $2.38 \mathrm{~cm}$ and covering a wedgeshaped region (shown in Figure 1) of approximately 1/12 of the area of the camera plate. Data from additional source locations were taken to verify the symmetry of the camera plate response.

The data for each $\gamma$-ray beam location were analyzed separately. After making preliminary cuts to select only photopeak events and to reject background events occurring outside the beam, the data for each beam were fit to a straight line in the space of PMT signals. The parameter $\lambda$ of distance along the line was defined such that $\lambda=0$ corresponded to $\gamma$-ray interactions near the front of the crystal, while $\lambda=1$ corresponded to events at the back of the crystal, nearest the PMTs.

Thus, the normalized response functions determined from the beam measurements were expressed as functions of a depth parameter $\lambda$, rather than the physical depth $z$. We found that the depth parameter $\lambda$ is a non-linear function of $z$ and that the relation between $\lambda$ and $z$ varies as a function of the lateral $(\mathrm{x}, \mathrm{y})$ position. However, since our primary interest is in an optimum algorithm for the lateral position $(\mathrm{x}, \mathrm{y})$ we substituted $\lambda$ for $z$ in the maximum likelihood formulation and below we will refer to the event location $\vec{x}=(x, y, \lambda)$. 


\section{Position Algorithm}

While the details of the method by which the likelihood function is maximized are not critical to the results, we include for completeness a discussion of the particular approach we used.

The maximization of the likelihood function can be done by an iterative technique where a first guess $\vec{x}^{0}$ of the position is assumed to be close to the position $\vec{x}=\vec{x}^{0}+\Delta \vec{x}$ of the maximum. The displacement $\Delta \vec{x}$ is estimated by setting

$$
\frac{\partial \ln \left(\hat{L}\left(\vec{x}^{0}+\Delta \vec{x}\right)\right)}{\partial x_{k}} \approx \frac{\partial \ln \left(\hat{L}\left(\vec{x}^{0}\right)\right)}{\partial x_{k}}+\sum_{l} \frac{\partial^{2} \ln \left(\hat{L}\left(\vec{x}^{0}\right)\right)}{\partial x_{k} \partial x_{l}} \Delta x_{l}=0 .
$$

The solution for $\Delta x_{l}$ is then used to compute a refined guess of the position, and the procedure iterated.

Our algorithm approximates this procedure in two ways. First the positions $\vec{x}^{0}$ are restricted to the lattice of points at which we have measured the PMT responses (or inferred the responses by symmetry). Second, we solve equation [7] for $\Delta x_{l}$ with the assumption that deviations of the PMT responses $\hat{n}_{i}$ from the mean responses $\hat{N}_{i}\left(\vec{x}^{0}\right)$ are small. The resulting solution is:

$$
\Delta x_{l}=\sum_{i} X_{l i}\left(\hat{n}_{i}-\hat{N}_{i}\left(\vec{x}^{0}\right)\right)
$$

where

$$
X_{l i}=\sum_{k} \sigma_{l k} \frac{\partial \ln \left(\hat{N}_{i}\right)}{\partial x_{k}}
$$

and

$$
\left(\sigma^{-1}\right)_{l k}=\sum_{i} \frac{1}{\hat{N}_{i}} \frac{\partial \hat{N}_{i}}{\partial x_{k}} \frac{\partial \hat{N}_{i}}{\partial x_{l}}
$$

with all evaluations performed at $\vec{x}^{0}$.

The required derivatives of the normalized response functions $\hat{N}_{i}$ were computed by finite differences on the lattice, and for each lattice point we stored $\hat{N}_{i}$ and $X_{l i}$. Then equation [8] defined a linear algorithm valid for calculating the event position near $\vec{x}^{0}$. At a moderate distance $(-2-3 \mathrm{~cm})$ from this point distortions appeared as a compression or expansion of the $\mathrm{x}$ and $\mathrm{y}$ axes with increasing depth. To correct for this distortion we introduced a quadratic correction :

$$
\Delta x_{l}^{\prime}=\Delta x_{l}+\sum_{m n} C_{l m n} \Delta x_{m} \Delta x_{n}
$$

where

$$
C_{l m n}=-1 / 2 \sum_{i} X_{l i} \frac{\partial^{2} N_{i}}{\partial x_{m} \partial x_{n}}
$$

\section{Position and Energy Resolution Results}

Figure 1 shows measured event positions obtained by the likelihood analysis for collimated $662 \mathrm{keV} \gamma$-rays incident at a number of grid positions. Data were actually obtained at the grid points enclosed by the dashed curve and are repeated in Figure 1 by symmetry to illustrate the position resolution in relation to the diameter of the PMTs and the extent of the usable area of the camera plate. The usable area has a diameter of approximately $27 \mathrm{~cm}$. Outside this diameter the position resolution degrades due to edge effects.

The position resolution obtained at the center of the camera plate is illustrated in Figures 3 and 4 at $\gamma$-ray energies of $662 \mathrm{keV}$ and $122 \mathrm{keV}$ respectively. Since at $122 \mathrm{keV}$ the position broadening is due almost entirely to photo-electron statistics, the distribution of measured positions seen in Figure 4 is approximately Gaussian. In contrast, the distribution of measured positions obtained at

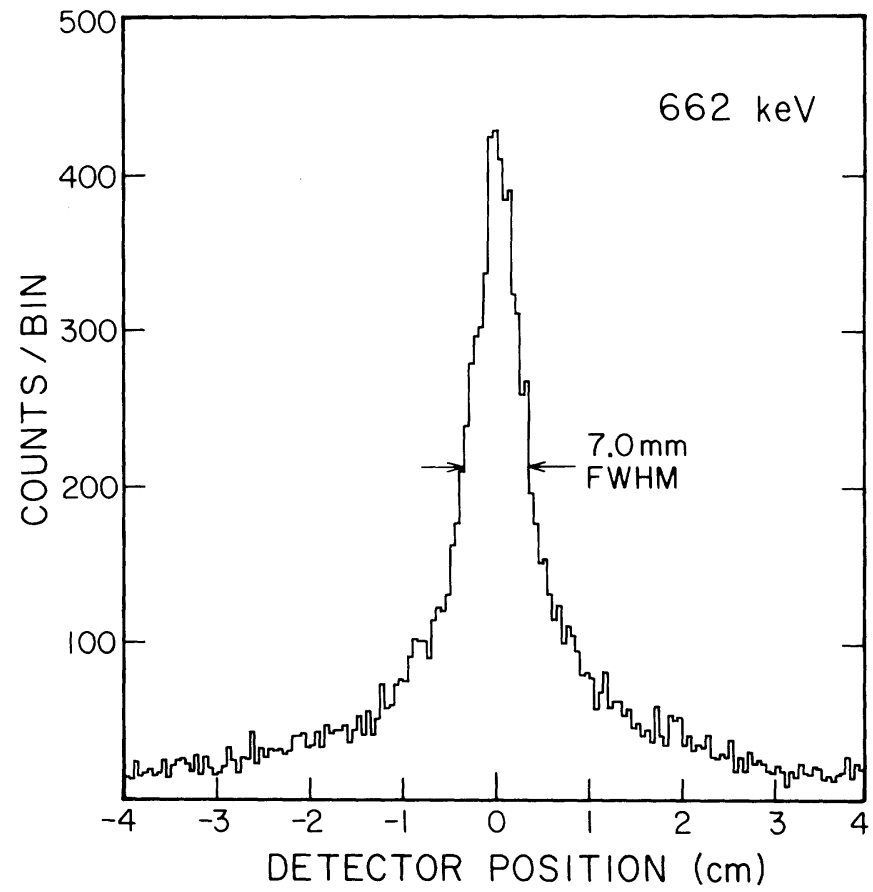

Figure 3. Histogram showing position resolution at $662 \mathrm{keV}$. If we subtract in quadrature the contribution due to the beam spread of $3 \mathrm{~mm}$ FWHM, we obtain a position resolution of $6.3 \mathrm{~mm}$ FWHM.

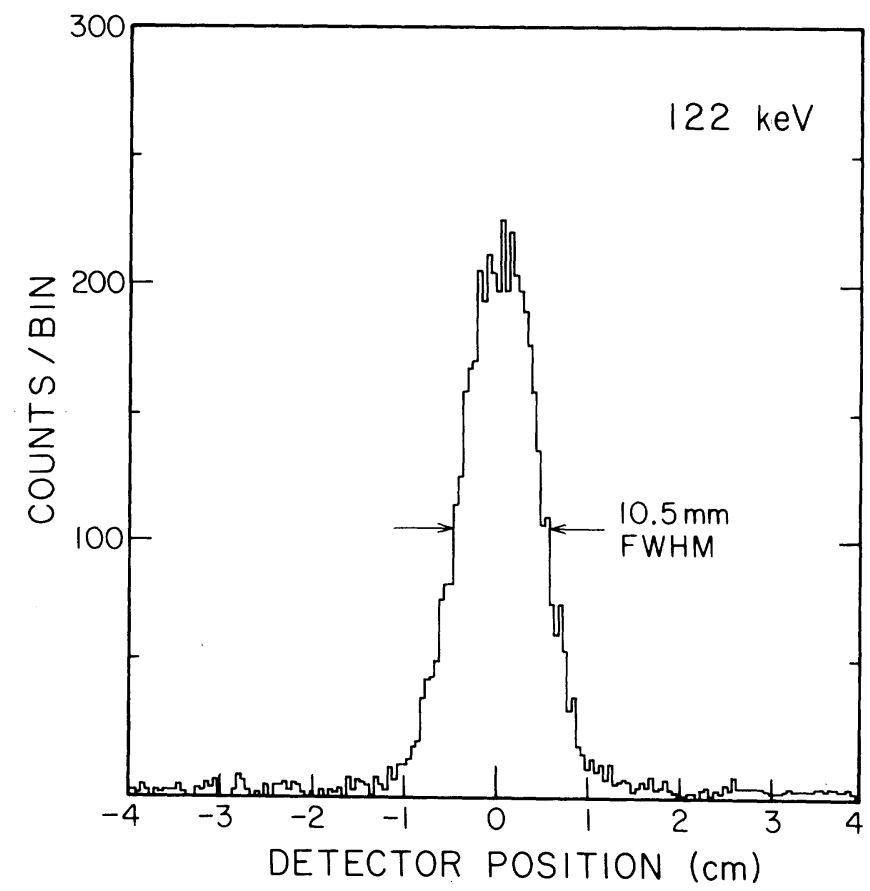

Figure 4. Histogram showing position resolution at $122 \mathrm{keV}$.

$662 \mathrm{keV}$ is distinctly non-Gaussian, with extended tails due to Compton scattering.

Figure 5 shows a calculation of the expected position resolution as a function of energy. The calculation employed a Monte Carlo $\gamma$-ray propagation code to determine the contribution to the FWHM position resolution due to Compton scattering which becomes important above an energy of approximately $300 \mathrm{keV}$. The Gaussian contribution due to photo-electron statistics and light collection effects, was normalized to the measured position resolution at $122 \mathrm{keV}$ where the effect of Compton scattering is negligible. The calculated curve agrees well with the data point at 662 $\mathrm{keV}$ which has been adjusted down from 7.0 to $6.3 \mathrm{~mm}$ to account 


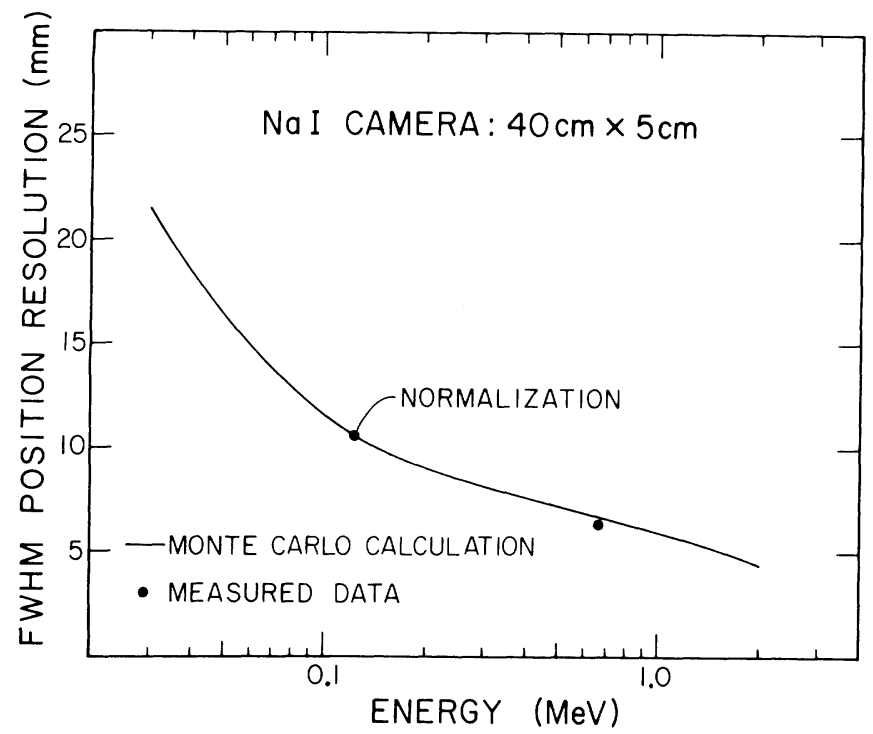

Figure 5. Comparison of FWHM position resolution measurements and results of a Monte Carlo calculation which accounts for Gaussian broadening due to photo-electron statistics and the non-Gaussian effect of Compton scattering. for the collimated beam width of $3.0 \mathrm{~mm}$ FWHM. Figure 5 indicates that in spite of Compton scattering the expected FWHM position resolution continues to improve up to the limit of the calculation at an energy of $2 \mathrm{MeV}$.

While we have yet to measure the energy resolution of the camera plate over a large area, the resolution measured at the center of the detector was 7\% FWHM at $662 \mathrm{keV}$.

\section{Depth Discrimination and Background Reduction}

A consequence of the development of a three dimensional position determination algorithm is the capability of measuring the depth of the $\gamma$-ray interaction in the detector. This depth determination capability is potentially very useful since it allows a reduction of background. For example, low energy $\gamma$-rays are expected to interact primarily near the front face of the crystal. The interaction length for $\gamma$-rays of less than $300 \mathrm{keV}$ energy is less than $1.7 \mathrm{~cm}$. Thus, low energy events with interaction positions in the back half of the Anger camera can be rejected as background induced events. Furthermore, the rejection criteria can be optimized for the energy of interest since the depth determination is performed ex post facto in the data analysis.

Figure 6 gives an indication of the depth determination capability of the camera plate. The detector was flooded from front and back by $\gamma$-rays of $122 \mathrm{keV}$. Figure 6 shows the individual histograms of the depth parameter $\lambda$ for events incident from the front and back. A depth selection which accepts $90 \%$ of the $122 \mathrm{keV} \gamma$ rays incident from the front will reject $87 \%$ of the $\gamma$-rays incident from the back. The same depth selection will reduce a uniform internal background by a factor of two.

The background rejection provided by the depth selection will improve the flux sensitivity of the detector. The extent of this improvement will depend on the exact spatial distribution of background observed during a measurement.

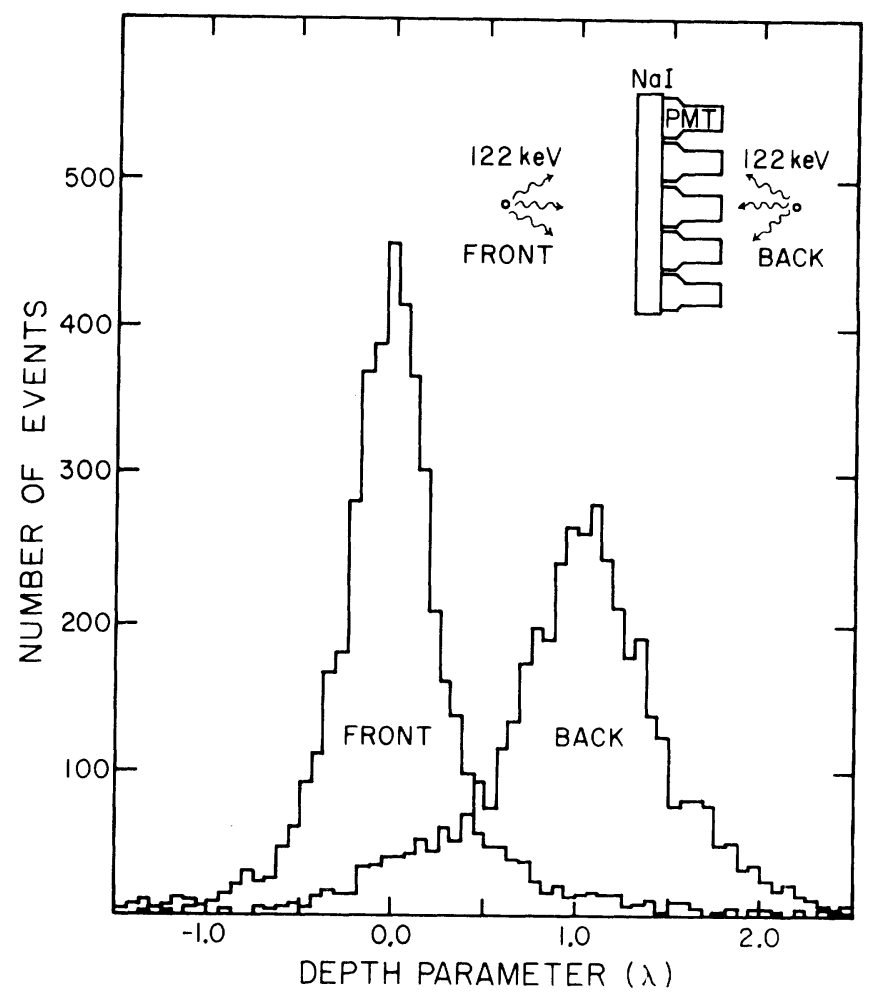

Figure 6. Separate histograms of the depth parameter $(\lambda)$ obtained for back and front incident uncollimated $122 \mathrm{keV}$ $\gamma$-rays.

\section{Implications for Gamma-Ray Telescopes}

The thick $\mathrm{NaI}(\mathrm{Tl})$ Anger camera has a variety of applications as a position sensitive detector for $\gamma$-ray imaging telescopes. Specifically, the good efficiency and position resolution over a wide energy range make the thick camera plate a suitable component of coded aperture and modulation collimator $\gamma$-ray imaging systems.

When combined with a properly designed coded aperture the thick $\mathrm{NaI}(\mathrm{Tl})$ camera plate discussed in this paper will become part of a first generation balloon-borne imaging $\gamma$-ray telescope. The detector's position resolution determines the telescope's angular resolution through its direct influence on the choice of the coded aperture cell width $w$. Using the formulae presented in reference [2] we have determined that a cell width of $2.5 \mathrm{~cm}$ will provide a good match to the camera plate position resolution of $<1.0 \mathrm{~cm}$ FWHM. With this choice of mask cell width the telescope will achieve both good flux sensitivity and angular resolution. With the coded aperture placed at a separation distance $L$ of 2.5 meters from the detector, as is feasible for a balloon-borne telescope, the angular resolution is $w / L=0.5$ degrees.

Thick $\mathrm{NaI}(\mathrm{Tl})$ camera plates of the type discussed in this paper can also be used as the position sensitive detectors in modulation collimator systems. In these systems the photon source signal is spatially modulated by a pair of occulting grids, allowing a position sensitive detector to measure the Fourier components of the source angular distribution..$^{4,5}$ Very fine angular resolution is possible. An advanced space telescope employing modulation collimator grids with $0.3 \mathrm{~mm}$ pitch separated by $50 \mathrm{~m}$ would obtain an angular resolution of 1 arc second. 


\section{Acknowledgements}

This work was supported in part by NASA grant NGR 05-002160, and the Presidential Young Investigator Program, NSF grant AST-8351736. We thank Prof. E. Stone, W. E Althouse, A. Cummings, and the other members of the GRIP (Gamma-Ray Imaging Payload) team for their numerous contributions to this work.

\section{References}

[1] H. O. Anger, "Scintillation Camera", Rev. Sci. Instr., vol. 29, pp. 27-33, January 1958.

[2] W. R. Cook, M. Finger, T. A. Prince, and E. C. Stone, "Gamma-Ray Imaging With a Rotating Hexagonal Uniformly Redundant Array", IEEE Trans. Nucl. Sci., vol. NS-31, no. 1, pp. 771-775, February 1984.

[3] P. R. Bevington, Data Reduction and Error Analysis for the Physical Sciences, McGraw-Hill, New York, 1969, p. 111.

[4] K. Makishima, S. Miyamoto, T. Murakami, J. Nishimura, M. Oda, Y. Ogawara, and Y. Tawara, "Modulation Collimator as an Imaging Device", in New Instrumentation for Space Astronomy, ed: K. A. van der Hucht and G. Vaiana, Pergamon Press, New York, 1977, pp. 277-289.

[5] G. J. Hurford and H. S. Hudson, "Fourier-Transform Imaging for X-Ray Astronomy", (BBSO\#0180), (UCSD-SP-79-27), 1979. 